\title{
Antiphospholipid Syndrome Alliance for Clinical Trials and International Networking (APS ACTION): 10-Year Update
}

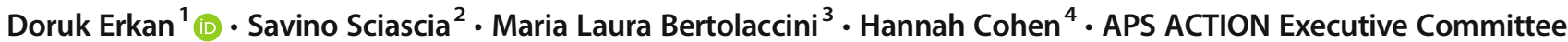

Accepted: 23 March 2021 / Published online: 1 May 2021

(C) The Author(s), under exclusive licence to Springer Science+Business Media, LLC, part of Springer Nature 2021, corrected publication 2021

\begin{abstract}
Purpose of Review APS ACTION is an international research network created to design and conduct large-scale, multicenter research in persistently antiphospholipid antibody (aPL)-positive patients. Given the expanding research activities of the network in the last decade since its creation, the purpose of this article is to review the scientific contributions of APS ACTION as well as future directions.

Recent Findings APS ACTION has achieved increased international collaboration with internal and external investigators for outcome, interventional, and mechanistic antiphospholipid syndrome (APS) studies. This has been linked to substantial progress in Core laboratory work, which has demonstrated that laboratories can achieve good agreement in performance of aPL assays by use of the same reagents, analyzer type, and protocols.

Summary APS ACTION will continue to identify gaps in the existing aPL/APS literature, design mechanistic studies to elucidate underlying mechanisms, and conduct prospective, large-scale clinical studies, all for the ultimate goal of early diagnosis and improved management of aPL-positive patients.
\end{abstract}

Keywords Antiphospholipid syndrome $\cdot$ Antiphospholipid antibodies $\cdot$ APS ACTION

\section{Introduction}

Antiphospholipid syndrome (APS) is an autoimmune disorder characterized by thrombosis and pregnancy morbidity in patients with persistently positive antiphospholipid antibodies (aPL). Antiphospholipid antibodies that are used for APS classification include lupus anticoagulant test (LA), anticardiolipin antibodies (aCL), and anti- $\beta_{2}$ glycoprotein-I antibodies ( $\mathrm{a} \beta_{2}$ GPI) [1].

Antiphospholipid Syndrome Alliance for Clinical Trials and International Networking (APS ACTION) is an international research network created to design and conduct largescale, multicenter research in persistently aPL-positive

This article is part of the Topical Collection on Antiphospholipid Syndrome

Doruk Erkan

erkand@hss.edu

Extended author information available on the last page of the article patients. The network is composed of multidisciplinary physicians, scientists, and investigators from around the world interested in APS research. The founding principle of APS ACTION is an international collaborative effort, open to qualified investigators across the globe who are committed to furthering our understanding of APS and its management.

Given the expanding research activities of the network in the last decade since its creation, the purpose of this article is to review the scientific contributions of APS ACTION as well as future directions.

\section{0: The Inception of APS ACTION}

The International Congress on aPL is held every three years to discuss the recent advances and future directions in APS. The Antiphospholipid Syndrome Clinical Research Task Force (APS CRTF) was one of the task forces developed by the "13th International Congress on aPL" (2010) organization committee with the purpose of (a) evaluating the limitations of APS clinical research and (b) developing guidelines for 
researchers to help improve the quality of APS research [2]. The core recommendation of the APS CRTF was that there is an urgent need for a true international collaborative approach to design and conduct well-designed, prospective, large-scale, multicenter clinical trials and research of patients with persistent and clinically meaningful aPL profiles. Thus, an international collaborative working summit took place in Miami, FL, USA (November 2-4, 2010), that resulted in the formation of a clinical trial research alliance entitled APS ACTION (www. apsaction.org) [3].

\section{0-2020: The Evolution of APS ACTION}

The network currently comprises 66 multidisciplinary physicians and investigators interested in APS research from 38 international centers (see Appendix). The APS ACTION Executive Committee, co-chaired by Drs. Maria Laura Bertolaccini, Hannah Cohen, and Doruk Erkan, comprises nine elected members, representing different regions of the world. Six APS ACTION Core Laboratories have been set up worldwide in Sao Paulo (Brazil, South America), Sapporo (Japan), Shanghai (China), Galveston (Texas, USA), Padova (Italy, Europe), and London (UK). APS ACTION Annual Summits foster international collaboration and facilitate discussions of the ongoing projects. As part of an effort to attract young talent to APS research, an APS ACTION Young Scholar Program has been established in which an annual award is distributed to recognize junior physician scientists who have contributed to APS research. The APS ACTION Young Scholar Exchange Program is designed to incentivize young physicians and/or scientists to get involved in APS-related basic or clinical research in one of the network centers; the goal is to increase their integration to our community and by enhancing their connections. The most recent initiative of the network, APS ACTION Scientific Interaction, is a virtual research progress meeting every two months to discuss ongoing projects. In addition, APS ACTION continues to work closely with APS patient organizations to improve awareness about the disease.

\section{What Did We Learn from APS ACTION Collaborative Research?}

\section{Literature Reviews}

The epidemiology of APS in the general population is not well established. Based on recent estimations, the incidence of APS is approximately $1-2$ cases per 100,000 persons per year, and the prevalence approximately ranges from 17 to 50 cases per 100,000 persons $[4,5]$. However, the true frequency of
aPL in different clinical settings is yet to be fully elucidated. In order to contribute to the understanding of the epidemiology of APS, APS ACTION researchers systematically reviewed the literature in early 2010 s analyzing 120 studies. Frequencies of aPL positivity in general-population patients with pregnancy morbidity (PM), deep vein thrombosis (DVT), myocardial infarction (MI), and stroke (ST) were $6 \%, 10 \%, 11 \%$, and $14 \%$ respectively $[6,7]$. A sub-analysis based on 81 studies considering all the outcomes together showed that the frequency of aPL positivity was threefold higher in patients, compared to controls (10\% vs. $3 \%)$. A follow-up study focusing on younger adults (less than 50 years old) estimated the frequency of aPL positivity in younger patients with stroke as $17 \%$. The presence of aPL conferred a fivefold higher risk for cerebrovascular events when compared with controls [8]. These reviews also emphasized the high degree of heterogeneity of the literature regarding type of aPL tests, definitions of aPL positivity, and clinical manifestations.

\section{APS ACTION Clinical Database and Repository ("Registry")}

The APS ACTION clinical database and repository ("Registry"), launched in 2012, is designed to investigate the natural course of persistently aPL-positive patients with or without autoimmune diseases over at least 10 years. The Registry represents a unique opportunity to perform large-scale, cross-sectional, and prospective analyses, which will eventually guide us to better understand the clinical features of APS patients who are followed annually with clinical data and blood collection. Selected recent analyses performed based on the APS ACTION registry data are discussed below.

The baseline characteristics of aPL-positive patients enrolled in the international registry have been described [9], focusing on overall features and clinical and laboratory subtypes. At the time of the analysis in 2019 , more than 800 aPLpositive patients (mean age: $45 \pm 13$ years; female: $74 \%$; white $68 \%$ ) were included. About one-third of patients had concomitant systemic autoimmune disease, approximately $70 \%$ of patients had thrombosis, and $50 \%$ of those with pregnancy history had obstetric morbidity. Interestingly, more than half of aPL-positive patients had at least one non-criteria manifestation. When focusing on the aPL profile among the $660 \mathrm{pa}-$ tients with three aPL tested, $42 \%$ presented with triple aPL positivity. While single, double, and triple aPL-positive subgroups had similar frequencies of vascular, obstetric, and noncriteria manifestations, within single aPL positivity, LA test positivity represented the major determinant of clinical manifestations (less frequently observed in the single aPL subgroup of $\mathrm{a} \beta_{2}$ GPI or aCL only). The analysis of the baseline characteristics confirmed the degree of heterogeneity among aPL- 
positive patients both in terms of clinical manifestations and laboratory profiles.

Another analysis investigating the impact of systemic lupus erythematosus (SLE) on the clinical phenotype of aPLpositive patients [10] observed that the frequency of some clinical manifestations, such as thrombocytopenia and hemolytic anemia, were higher in patients with SLE, when compared to patients without SLE. Similarly, patients with SLE more frequently developed low complement levels and IgA $\mathrm{a} \beta_{2}$ GPI. Conversely, cognitive dysfunction and IgG a $\beta_{2}$ GPI were observed more frequently in patients without SLE. Interestingly, the rates of arterial and venous events (including the frequency of recurrent thromboses), as well as pregnancy morbidity, did not significantly differ in patients with and without SLE. The prevalence of cardiovascular disease (CVD) risk factors at the time of entry did not seem to be influenced by the presence of SLE. Only smoking habits were observed more frequently in aPL-positive patients with SLE.

To further investigate the characteristics of aPL-positive patients, a hierarchical cluster analysis was conducted on the first 500 registry patients aiming to identify different clinical phenotypes [11]. Three main exclusive clusters were identified: (a) female patients with no other autoimmune diseases but with venous thromboembolism (VTE) and triple aPL positivity; (b) female SLE patients with positive LA and VTE, aPL nephropathy, thrombocytopenia, hemolytic anemia; and (c) older men with arterial thrombosis, heart valve disease, livedo, skin ulcers, neurological manifestations, and CVD risk factors. These innovative descriptive strategies again confirmed the heterogeneity of aPL-positive patients. Additionally, these strategies have paved the way for further translational research to understand disease mechanisms, design new approaches for APS classification, and ideally guide more tailored management.

Recently, longitudinal data from the registry were investigated to assess the stability over time of clinically meaningful aPL profiles, defined as positive LA test, aCLIgG/M $>40 \mathrm{U}$, and/or a $\beta_{2}$ GPI IgG/M > 40U [12]. More than 470 patients with clinically meaningful aPL profiles at baseline with a median follow-up of 5.1 years were analyzed. Approximately $80 \%$ patients had stable aPL profiles over time (defined as clinically meaningful profile in at least two-thirds of follow-up aPL measurements), while significant changes were observed over time in $11 \%$. Interestingly, time did not seem to significantly impact the probability of maintaining a clinically meaningful aPL profile. Furthermore, changes in the aPL profile were less frequently observed in patients with LA or triple aPL positivity when compared other aPL profiles. These findings will be of great value in upcoming validation studies of stored blood samples through APS ACTION core laboratories.

Based on additional registry analyses completed over the last decade, APS ACTION investigators demonstrated that (a) the adjusted global APS Score (aGAPSS) might help risk stratifying patients based on the likelihood of developing recurrent thrombosis in APS [13]; (b) younger age at diagnosis of obstetric APS, additional CVD risk factors, history of superficial vein thrombosis and heart valve disease, multiple aPL test positivity, and higher aGAPSS at the registry entry increase the risk of thrombosis after initial pregnancy morbidity [14]; and (c) combined antiplatelet and anticoagulant therapy, compared to single therapy, may decrease the rate of and increase the time to thrombosis recurrence in patients with APS presenting with arterial thrombosis [15].

\section{Core Laboratory Validation and Antiphospholipid Antibody Testing Standardization}

Variability remains a challenge in aPL testing. The APS ACTION Core laboratory validation exercises were designed to assess intra-laboratory and inter-laboratory variability (LA test, aCL, and $\mathrm{a} \beta_{2} \mathrm{GPI}$ ) in order to establish whether an acceptable degree of agreement among the APS ACTION Core laboratories could be achieved. The LA assay was validated based on the First International Reference Panel for LA (National Institute for Biological Standards and Control [NIBSC], UK) (negative, moderately positive, and strongly positive samples used by the Core laboratories) [16]. Anticardiolipin antibody and a $\beta_{2}$ GPI enzyme-linked immunoassays (ELISA) were validated based on blinded serum samples from low, medium, and high aPL-positive patients and from negative controls. After the completion of the validation exercises, registry samples were tested at Core laboratories. Two important studies were undertaken to examine the agreement in aPL results between APS ACTION Core laboratories (aPL tested based on validated protocols) and local laboratories (aPL results entered by the investigators).

For LA testing, five Core laboratories used the same reagents, analyzer type, protocols, and characterized samples for LA validation [17]. Non-anticoagulated registry samples were retested at the corresponding regional Core laboratories and anticoagulated samples at a single Core laboratory (UK). Clotting times for the reference/characterized plasmas used for normalized ratios were similar between Core laboratories (CV $<4 \%$ ); precision and agreement for LA positive/negative plasma were similar (all $\mathrm{CV} \leq 5 \%$ ) in the four laboratories that completed both parts of the validation exercise; 418 registry samples underwent LA testing. Agreement for LA positive/ negative status between Core and local laboratories was observed in $87 \%(115 / 132)$ of non-anticoagulated and $77 \%$ $(183 / 237)$ of anticoagulated samples. However, 29\% (120/ 418) of samples showed discordance between the Core and local laboratories or equivocal LA results. Some of the results of the local laboratories might have been unreliable in $25 \%$ $(41 / 166)$ and $23 \%(58 / 252)$ of the total non-anticoagulated and anticoagulated samples, respectively. Equivocal results by the Core laboratory might have also contributed to 
discordance. In conclusion, this study showed that APS ACTION Core laboratories can achieve good agreement in LA performance by use of the same reagents, analyzer type, and protocols.

For aPL ELISA assays, to assess the level of agreement between the Core and local laboratory aCL and a $\beta_{2}$ GPI ELISA results, 497 registry samples underwent confirmatory aPL tests following the validation exercise [18]. Categorical agreement between the core and local laboratory values, as expressed by Cohen's kappa coefficients, ranged between 0.61 and 0.80 (as substantial agreement). The correlation between quantitative results in the aCL and $\mathrm{a} \beta_{2}$ GPI was better for IgM and IgA compared to IgG (Spearman rho 0.789 and 0.666 vs. 0.600 for aCL and rho 0.892 and 0.744 vs. 0.432 for $\mathrm{a} \beta_{2}$ GPI). In conclusion, the results of inclusion for aCL and $\mathrm{a} \beta_{2}$ GPI tests used for recruitment into the registry were in agreement to the results obtained by the APS ACTION Core laboratories; aCL and a $\beta_{2}$ GPI results showed very good categorical agreement. This agreement increased when considering high titer (>40 units) samples.

\section{Clinical Trials}

The first major clinical trial designed by APS ACTION was the international, multicenter, randomized controlled trial (RCT) assessing the efficacy of hydroxychloroquine (HCQ) for primary thrombosis prevention in persistently aPLpositive but thrombosis-free patients with no other systemic autoimmune diseases. The study, partially supported by New York Community Trust, was terminated earlier than the planned completion date due to the low patient recruitment rate exacerbated by the prolonged manufacturing shortage and price increase of HCQ. Given that a small number of patients with a relatively short follow-up were enrolled in this RCT, and no patients developed thrombosis, we were not able to accurately assess the effectiveness of HCQ for primary thrombosis prevention in persistently aPL-positive patients with no other systemic autoimmune diseases. The details of the study design, results, and challenges have been addressed elsewhere [19].

\section{What Are the Current APS ACTION Collaborative Studies?}

\section{Literature Reviews}

Given the recent reports of aPL-positive patients during severe COVID-19 [20] and potential clinical implications of COVID-19 for APS patients, an APS ACTION COVID-19 Working Group is working on guidance that will include mechanistic and clinical aPL and COVID-19-related discussions based on an extensive literature review as well as an agenda for future research. In addition, APS ACTION is participating in current international collaborative efforts to shed some light on the definition of aPL nephropathy $[21,22]$.

\section{APS ACTION Clinical Database and Repository ("Registry")}

The APS ACTION Registry now includes approximately 850 patients in whom the baseline clinical and aPL phenotype have been accurately characterized. Our current efforts aim to better stratify these patients based on laboratory and clinical characteristics, also analyze the long-term outcomes. The identification of aPL-positive patients by different laboratory profiles and clinical phenotypes has the potential to improve risk stratification with the final aim of guiding clinicians to better differentiate the patients, understand clinical outcomes, and eventually prevent the occurrence of new events.

Comprehensive laboratory phenotyping, including antiphosphatidylserine/prothrombin antibodies (aPS/PT), $a \beta_{2}$ GPI domain I and IV/V antibodies, anti-neutrophil extracellular trap (NET) antibodies, and anti-activated protein $\mathrm{C}$ resistance (aPCR)/anti-protein $\mathrm{C}(\mathrm{aPC})$ antibodies have been undertaken to better define the laboratory characteristics of the APS ACTION registry cohort.

Selected registry-based outcome studies have been focusing on (a) longitudinal assessment of thrombosis risk [23] and pregnancy outcomes [24] in aPL-positive patients; (b) characteristics and outcomes of thrombotic APS patients not receiving of anticoagulation; (c) geographical clinical and laboratory differences among aPL-positive patients; and (d) the role of immunosuppression in primary APS.

\section{Core Laboratory Validation}

Lupus anticoagulant testing and detection, a critical step for accurate diagnosis and management of APS patients, is characterized by considerable variability between laboratories. Lupus anticoagulant assessment in samples from patients receiving oral anticoagulants such as warfarin and other vitamin $\mathrm{K}$ antagonists is particularly challenging, since most LA tests require the presence of vitamin $\mathrm{K}$-dependent coagulation factors [25]. Thus, there is concern about the validity of LA test results in anticoagulated samples because of the potential for false positive/negative results. This includes concern about samples from patients receiving direct oral anticoagulants (DOACs), as these agents (the factor Xa [FXa] inhibitors rivaroxaban, apixaban and edoxaban, and dabigatran, a direct thrombin inhibitor) may interfere with LA tests and may thus lead to erroneous results [26]. However, determination of LA status is required in certain clinical settings as well as for full characterization of aPL status of patients in research studies and registries such as APS ACTION.

As discussed above, when we compared Core and local laboratory results [16], we identified that the discordance in LA status was more prevalent in the anticoagulated samples $(23 \%$, 
54/237), when compared to non-anticoagulated ones (13\%, 17/ 132) $(p=0.03)$. This is of high clinical relevance as the true result, either positive or negative, could have a major impact on patient management. For this reason, a new project, supported by APS Support UK, aims (a) first, to establish a validated protocol for all APS ACTION Core laboratories to test for LA status in patients receiving anticoagulation; and (b) second, to test for LA status in the serial APS ACTION anticoagulated samples from follow-up visits up to 10 years. For the validation exercise, the APS ACTION Core Laboratories would use the same reagents, same LA-positive and negative control plasmas, under the same conditions and the same protocol. This study would provide a highly standardized, consistent assessment of LA status by the Core laboratories that is required to underpin the accurate interpretation of APS ACTION clinical data. It could also provide a protocol to contribute to working towards widespread international standardization of LA testing, to underpin accurate diagnosis and management of APS patients.

\section{Clinical Trials}

Following the early termination of our HCQ primary thrombosis prevention trial [19], APS ACTION has contributed to the site selection (by promoting the studies to members) of the completed, ongoing, or planned APS clinical trials initiated by APS ACTION members [27, 28]. A major current effort is directed at collaboratively designing multicenter clinical trials in aPL-positive patients.

\section{0 and Beyond: Time to Explore New Horizons}

The APS ACTION Registry has matured over the past 10 years, with an established infrastructure, and an active and co-operative collaboration of international multidisciplinary expert committed to the optimal care of patients. As evidenced by the preceding sections of this report and set out as a goal in our five-year update [29], APS ACTION has achieved increased collaboration with internal and external investigators for outcome, interventional, and mechanistic studies. This has been linked to substantial progress in Core laboratory work, which has demonstrated that laboratories can achieve good agreement in performance of aPL assays by use of the same reagents, analyzer type, and protocols $[17,18]$. These standardized Core laboratory results underpin the accurate interpretation of APS ACTION clinical data.

Since its creation, APS ACTION has developed an established track record, with achievements including active participation in major scientific meetings, many publications in peer-reviewed journals, and useful interactions with patients and patient organizations. However, while there have been significant advances in our understanding of APS, there still remains many questions without evidence-based answers. The ultimate goal of APS ACTION is to improve the care of APS patients, with cognizance of the wide clinical and laboratory heterogeneity, and now is the time to explore new horizons in working towards that goal.

\section{Gaps to Fill in the Literature}

Further advances in the pathophysiology, early diagnosis, and risk stratification of APS require a focus on aiming to fill the gaps in the literature. The first is definition of the prevalence of APS, which is of fundamental importance, as this has implications for the approach to clinical studies, and patient investigation and management. Patients with rare diseases might require national and international networks for the identification, classification, and study of rare diseases and for the education of affected patients. Matching research design to attributes of rare diseases and interventions can facilitate the completion of RCTs that are adequately powered [30].

Based on population studies, APS meets the definition of a rare disease as described by Holué (prevalence $\leq 5$ per 10,000 population) [31], with the reported prevalence $1.7-5$ per 10,000 population $[4,5,32]$. However, based on our systematic reviews discussed above [6-8] and other prospective studies $[33,34]$ the true prevalence of APS is difficult to calculate given the lack of large-scale well-designed population studies, and the relatively common challenge of APS underdiagnosis and overdiagnosis. Venous thromboembolism (VTE), stroke, and pregnancy morbidity are common conditions, and further studies are needed to define the true prevalence of APS in these patient populations, together with assessment of the potential clinical impact and cost implications of widespread screening for aPL.

Another unmet need is focused systematic reviews to inform therapeutic strategies/studies. An example of a systematic review that provided the basis for a clinically useful study was the review that concluded that although a positive aPL test appears to predict an increased risk of recurrence in patients with a first VTE, the strength of this association is uncertain because the available evidence is of very low quality [35]. A subsequent prospective cohort study, including the same authors, of aPL and recurrent thrombosis after a first unprovoked VTE demonstrated that aPL and D-dimer are independently associated with recurrence after a first unprovoked VTE [22].

\section{APS ACTION Clinical Database and Repository ("Registry")}

To date, with observational data covering almost the 10 -year follow-up, the registry can be considered a unique tool of its kind to perform prospective analyses in patients with aPL to 
address open questions related to APS. Future prospective analyses, using both standardized Core laboratory aPL tests and registry data, will guide future research aiming to investigate the role of aPL risk profiles and improve risk stratification. Furthermore, detailed characterization of the APS ACTION Registry cohort could provide a basis to explore the utility of approaches such as autoantigenomics, genomewide association studies (GWAS), and whole genome sequencing (WGS) for risk stratification and ultimately a precision medicine-based approach.

Risk stratification of APS patients can be based on the clinical and/or laboratory phenotype. From a clinical point of view, thrombotic recurrence risk may differ for patients with aPL who experience initial venous or arterial thrombotic events, demonstrated in a systematic review and meta-analysis [36]. The authors of this study suggested that prospective clinical trials are needed for different thrombotic phenotypes in patients with aPL; as also supported by our studies [11], cluster analysis provides a foundation to understand disease mechanisms, create new approaches to classification, and ultimately develop tailored management.

Risk stratification by laboratory criteria is currently based on criteria aPL tests; LA test carries the highest risk for thrombosis among all aPL tests [37] and the occurrence of a thrombotic event may be associated with higher mortality in patients with LA [38]. Triple aPL positivity is the aPL phenotype that confers the highest risk of thrombosis [39]. Non-criteria aPL, such as aPS/PT [40], anti-domain-1 $\mathrm{a} \beta_{2}$ GPI [41], anti-protein C/activated protein $\mathrm{C}$ resistance (APCr) [42], and anti-NET antibodies [43], also appear to be relevant with regard to thrombotic risk. An association between complement activation and recurrent thrombosis in APS patients has been demonstrated using a functional modified HAM (mHAM) assay, and patient-derived $\mathrm{a}_{2} \mathrm{GPI}$ also increased C5b-9 deposition on the cell surface [44]. Thus, assessment of the APS APCTION registry patient's proinflammatory profile may also contribute to risk stratification.

Some other recently introduced approaches may inform risk stratification of APS patients. Particle-based multi-analyte technology (PMAT), using particle-based multi-analyte testing, supports aPL panel algorithm testing. The APS reagents detect antibodies of IgG, IgA, and IgM isotypes to aCL, $\mathrm{a}_{2} \mathrm{GPI}$, and aPS/PT, resulting in a panel profile of nine different aPL tests. Particle-based multi-analyte technology holds the promise of closing serological gaps in autoantibody diagnostics in APS [45]. Autoantigenomics describes holistic characterization of an individual's autoantigen repertoire, involving identification of subgroups of patients who carry particular clusters of targeted autoantigens, e.g., belonging to signalling pathways, to identify potential therapeutic targets. Thus, novel single or panels of autoantigens can be employed as biomarkers for diagnosis/prognosis [46]. Precision medicine comprises a tailored approach to each patient, based on genetic and epigenetic singularities and drug response. An optimal approach requires initial evaluation to define the phenotype and subphenotype, assess the main pathphysiologic pathway through biomarkers, to predict potential outcomes, and inform the best choice of treatment and follow-up care, all leading to cost-effective outcomes. A precision medicine approach is being developed for SLE [47, 48] and requires application also to APS. Over the past decade, GWAS have successfully identified associations of thousands of singlenucleotide polymorphisms (SNPs) with human traits and diseases [49]. A recent study suggests that even when applying a GWAS approach to the study of inflammatory biomarkers, there is increased power and precision using WGS data, presumably due to more accurate determination of genotypes [50]. The APS ACTION Core laboratory network provides an established base to support a structured approach to the development of novel approaches to improve APS diagnosis and risk stratification.

\section{Clinical Trials}

Although developing well-designed multicenter clinical trials is an important goal of APS ACTION, an equally important need in the field of APS is development of a toolkit to support APS clinical studies. Components of toolkit include suitable outcome measures for clinical studies, i.e., optimal damage index, specific quality of life index, and disease activity index.

The initiation and development of appropriate clinical studies requires a focus on suitable outcome measures [51]. For instance, a damage index (DIAPS) has previously been proposed for thrombotic APS [52]. Of note, key manifestations of APS, such as livedo reticularis/racemosa, multiple sclerosislike disease, and diffuse pulmonary hemorrhage, are not included [53]. A retrospective analysis of 50 primary APS vs. 50 APS/SLE patients reported distinct patterns of damage in the two groups: damage in PAPS an early event, while APS/SLE associated with higher long-term damage, with striking increment of damage during follow-up [54]. An APS ACTION registry-based DIAPS study in ongoing; and similar studies should be conducted to help improve the quality of APS clinical trials.

Disease burden/damage, anticoagulation, and lack of social support contribute to the impaired health-related quality of life (HRQoL) in APS patients, measured by the SF-36 and EQ-5D [55]. In SLE patients, the LupusQoL disease-specific questionnaire is more sensitive and shows greater responsiveness to change than the generic SF-36 [56]. In addition, postthrombotic syndrome shows a significant impact on diseasespecific QoL score, not captured by the SF-36 [57]. An option to consider is to adapt the SF-36 for APS. The impact of the accrual damage and treatment effect/complications in APS patients and resultant impact on the HRQoL is not established. 
Assessment of disease activity index is challenging in APS as many thrombotic consequences result in permanent effects. However, some manifestations, such as transient ischemic attacks or the development of thrombocytopenia, which are amenable to treatment, can be considered as activity features. The time is surely right for a group of international experts to consider developing and testing a disease activity index.

\section{Conclusion}

Antiphospholipid syndrome remains a debilitating disease, with significant morbidity and mortality implications, particularly among previously healthy individuals. Over the past five years, APS ACTION has fulfilled an important need in APS research as the first international collaboration among APS clinicians and investigators focused on conducting multicenter, RCTs. We have grown substantially in terms of increasing membership worldwide, expanding our leadership group, and accumulating a large, international database of aPL-positive patients. In addition, tremendous progress has been made by our members towards facilitating international research collaboration and data sharing in order to advance APS research and development of Core laboratories with the goal of standardizing aPL testing. Members continue to identify gaps and limitations in the existing aPL/APS literature, which APS ACTION strives to improve upon through the design of prospective, large-scale studies with the goal of early diagnosis and risk stratification, increased basic science research to elucidate underlying mechanisms, and improved therapies and ultimately a cure, all hopefully facilitated by APS ACTION.

Development of the role of APS ACTION in contributing to optimal APS patient care requires a multitargeted approach. A focus is required on trying to define optimal antithrombotic strategies, and the use of the adjunctive modalities such as hydroxychloroquine, statins, and vitamin $\mathrm{D}$, as well as immunomodulation, complement inhibition, and potential new players. Where possible, systematic reviews utilizing data from the APS ACTION Registry and associated laboratory studies could lead to prospective cohort studies using standardized management based on guidelines and consensus, while RCT data remains limited. The initiation and development of appropriate clinical studies necessitates the development of suitable outcome measures, including a disease activity index, an optimal damage index, and a specific quality of life index. Progress in clinical studies should be linked to parallel development of clinical and laboratory-based risk stratification, and translational and basic research to increase our understanding of pathophysiological mechanisms. In addition, APS ACTION is now well placed to explore the development of a precision medicine approach to optimize APS patient care, which should include investigation of the use of autoantigenomics, GWAS, and WGS. Successful progression of the APS ACTION initiative requires a structured program of work and funding strategies, to maintain the infrastructure and to support clinical and laboratory studies, with continued development of partnerships with industry. Finally, APS ACTION will maintain close links with patients, with the ultimate goal optimization of patient care and experience.

Acknowledgements This article is prepared on behalf of the APS ACTION Executive Committee. In addition to the authors, APS ACTION Executive Committee Members include: Danieli Andrade (University of Sao Paulo, Sao Paulo, Brazil), Tatsuya Atsumi (University of Hokkaido, Sapporo, Japan), Maria Gerosa (University of Milan, Milan, Italy), Michelle Petri (Johns Hopkins University School of Medicine, Baltimore, MD, USA), Robert Roubey (University of North Carolina, Chapel Hill, NC, USA), and Maria Tektonidou (National and Kapdistrian University of Athens, Athens, Greece).

APS ACTION Members: Argentina: Santa Fe (Guillermo Pons-Estel); Australia: Sydney (Bill Giannakopoulos, Steve Krilis); Brazil: Rio de Janeiro (Guilherme de Jesus, Roger Levy), São Paulo (Gustavo Balbi, Danieli Andrade); Canada: Calgary (Ann Clarke, Leslie Skeith), Quebec (Paul F. Fortin); China: Beijing (Lanlan Ji, Zhouli Zhang), Shanghai (Hui Shi, Chengde Yang); France: Nancy (Stephane Zuily, Denis Wahl); Greece: Athens (Maria Tektonidou); Italy: Brescia (Cecilia Nalli, Laura Andreoli, Angela Tincani), Milan (Cecilia B. Chighizola, Maria Gerosa, Pierluigi Meroni), Padova (Chunyan Cheng, Alessandra Banzato, Vittorio Pengo), Turin (Silvia Foddai, Massimo Radin, Savino Sciascia); Jamaica: Kingston (Stacy Davis); Japan: Sapporo (Olga Amengual, Tatsuya Atsumi); Lebanon: Beirut (Imad Uthman); Netherlands: Utrecht (Maarten Limper, Philip de Groot); Spain: Barakaldo (Guillermo Ruiz Irastorza, Amaia Ugarte), Barcelona (Jose Pardos-Gea, Ignasi Rodríguez-Pinto, Roberto Ríos-Garcés, Ricard Cervera), Madrid (Esther Rodriguez, Maria Jose Cuadrado), Cordoba (Maria Angeles Aguirre Zamorano, Rosario Lopez-Pedrera); Turkey: Istanbul (Bahar Artim-Esen, Murat Inanc); United Kingdom: London (Maria Laura Bertolaccini, Hannah Cohen, Maria Efthymiou, Munther Khamashta, Ian Mackie, Giovanni Sanna); USA: Ann Arbor (Jason S. Knight), Baltimore (Michelle Petri), Boston (Rebecca Karp-Leaf), Chapel Hill (Robert Roubey), Durham (Tom Ortel), Galveston (Emilio Gonzalez, Rohan Willis), New Hyde Park (Nina Kello), New York City (H. Michael Belmont, Steven Levine, Jacob Rand, Medha Barbhaiya, Doruk Erkan, Jane Salmon, Michael Lockshin), Rochester (Ali Duarte), Salt Lake City (D. Ware Branch).

Funding The APS ACTION registry was created using REDCAP provided by the Clinical and Translational Science Center at Weill Cornell Medical College (CTSC grant UL1 TR000457). APS ACTION has received partial support from a private donor, APS Foundation of America, APS Support UK, and APS Foundation of Australia. Antiphospholipid antibody kits used at Core laboratories have been donated by INOVA Diagnostics and Instrumentation Laboratory. ML Bertolaccini is supported by the King's BHF Centre for Award Excellence RE/18/2/34213.

\section{Declarations}

Human and Animal Rights and Informed Consent This article does not contain any studies with human or animal subjects performed by any of the authors.

Conflict of Interest The authors declare no competing interests. 


\section{References}

1. Miyakis S, Lockshin MD, Atsumi T, Branch DW, Brey RL, Cervera $\mathrm{R}$, et al. International consensus statement on an update of the classification criteria for definite antiphospholipid syndrome. J Thromb Haemost. 2006;4(2):295-306.

2. Erkan D, Derksen R, Levy R, Machin S, Ortel T, Pierangeli S, et al. Antiphospholipid syndrome clinical research task force report. Lupus. 2011;20(2):219-24.

3. Erkan D, Lockshin MD, APS ACTION members. APS ACTIONAntiPhospholipid Syndrome Alliance for clinical trials and international networking. Lupus. 2012;21(7):695-8.

4. Duarte-García A, Pham MM, Crowson CS, Amin S, Moder KG, Pruthi RK, et al. The epidemiology of antiphospholipid syndrome: a population-based study. Arthritis Rheum. 2019;71(9):1545-52.

5. Radin M, Sciascia S, Bazzan M, Bertero T, Carignola R, Montabone E, et al. Antiphospholipid syndrome is still a rare disease-estimated prevalence in the Piedmont and Aosta Valley regions of Northwest Italy: comment on the article by DuarteGarcía et al. Arthritis Rheum. 2020;72(10):1774-6.

6. Chighizola CB, Andreoli L, de Jesus GR, Banzato A, Pons-Estel GJ, Erkan D, et al. The association between antiphospholipid antibodies and pregnancy morbidity, stroke, myocardial infarction, and deep vein thrombosis: a critical review of the literature. Lupus. 2015;24(9):980-4.

7. Andreoli L, Chighizola CB, Banzato A, Pons-Estel GJ, Ramire de Jesus G, Erkan D. Estimated frequency of antiphospholipid antibodies in patients with pregnancy morbidity, stroke, myocardial infarction, and deep vein thrombosis: a critical review of the literature. Arthritis Care Res. 2013;65(11):1869-73.

8. Sciascia S, Sanna G, Khamashta MA, Cuadrado MJ, Erkan D, Andreoli L, et al. The estimated frequency of antiphospholipid antibodies in young adults with cerebrovascular events: a systematic review. Ann Rheum Dis. 2015;74(11):2028-33.

9. Sevim E, Zisa D, Andrade D, Sciascia S, Pengo V, Tektonidou MG, et al. Characteristics of antiphospholipid antibody positive patients in AntiPhospholipid Syndrome Alliance for Clinical Trials and InternatiOnal Networking. Arthritis Care Res. 2020. https://doi.org/10.1002/acr.24468 Online ahead of print.

10. Unlu O, Erkan D, Barbhaiya M, Andrade D, Nascimento I, Rosa R, et al. The impact of systemic lupus erythematosus on the clinical phenotype of antiphospholipid antibody-positive patients: results from the AntiPhospholipid Syndrome Alliance for clinical trials and InternatiOnal Clinical Database and Repository. Arthritis Care Res. 2019;71(1):134-41.

11. Zuily $\mathrm{S}$, Clerc-Urmès I, Bauman $\mathrm{C}$, Andrade $\mathrm{D}$, Sciascia $\mathrm{S}$, et al. Cluster analysis for the identification of clinical phenotypes among antiphospholipid antibody-positive patients from the APS ACTION Registry. Lupus. 2020;23:961203320940776.

12. Gkrouzman E, Sevim E, Finik J, Andrade D, Pengo V, et al. Antiphospholipid antibody profile stability over time: prospective results from APS ACTION Clinical Database and Repository. J Rheumatol. 2020:jrheum.200513.

13. Radin M, Sciascia S, Erkan D, Pengo V, Tektonidou MG, Ugarte A, et al. The adjusted global antiphospholipid syndrome score (aGAPSS) and the risk of recurrent thrombosis: results from the APS ACTION cohort. Semin Arthritis Rheum. 2019;49(3):464-8.

14. de Jesús GR, Sciascia S, Andrade D, Barbhaiya M, Tektonidou M, Banzato A, et al. Factors associated with first thrombosis in patients presenting with obstetric antiphospholipid syndrome (APS) in the APS Alliance for Clinical Trials and International Networking Clinical Database and Repository: a retrospective study. BJOG. 2019;126(5):656-61.

15. Jackson WG, Oromendia C, Unlu O, Erkan D, DeSancho MT, Antiphospholipid Syndrome Alliance for Clinical Trials and
International Networking. Recurrent thrombosis in patients with antiphospholipid antibodies and arterial thrombosis on antithrombotic therapy. Blood Adv. 2017;1(25):2320-4.

16. Mackie IJ, Pengo V, Gray E, et al. Antiphospholipid Syndrome Alliance for Clinical Trials and International Networking core laboratory validation exercise: an international performance assessment of lupus anticoagulant (LA) tests using standardized methods and reagents. J Thromb Haemost. 2015;13(Suppl 2):777 (abstract).

17. Efthymiou M, Mackie IJ, Lane PJ, Andrade D, Willis R, Erkan D, et al. Comparison of real world and core laboratory lupus anticoagulant results from the Antiphospholipid Syndrome Alliance for Clinical Trials and International Networking (APS ACTION) clinical database and repository. J Thromb Haemost. 2019;17(12):2069-80.

18. Sciascia S, Willis R, Pengo V, Krilis S, Andrade D, Tektonidou $\mathrm{MG}$, et al. The comparison of real world and core laboratory antiphospholipid antibody ELISA results from antiphospholipid syndrome alliance for clinical trials \& international networking (APS ACTION) clinical database and repository analysis. Thromb Res. 2019;175:32-6.

19. Erkan D, Unlu O, Sciascia S, Belmont HM, Branch DW, Cuadrado MJ, et al. Hydroxychloroquine in the primary thrombosis prophylaxis of antiphospholipid antibody positive patients without systemic autoimmune disease. Lupus. 2018;27(3):399-406.

20. Gkrouzman E, Barbhaiya M, Erkan D, Lockshin MD. Reality check on antiphospholipid antibodies in COVID-19-associated coagulopathy. Arthritis Rheum. 2020. https://doi.org/10.1002/art.41472.

21. Taghavi M, Barbhaiya M, Tektonidou M, Fortin P, Andrade D, et al. Descriptive analysis of biopsy-proven antiphospholipid antibodyassociated nephropathy patients included in the AntiPhospholipid Syndrome Alliance for Clinical Trials and InternatiOnal Networking (APS ACTION) Clinical Database and Repository ("Registry") [abstract]. Arthritis Rheumatol. 2019; 71 (suppl 10). https://acrabstracts. org/abstract/descriptive-analysis-of-biopsy-proven-antiphospholipidantibody-associated-nephropathy-patients-included-in-theantiphospholipid-syndrome-alliance-for-clinical-trials-andinternational-networking-aps/. Accessed December 25, 2020.

22. Barbhaiya M, Erkan D, Zuily S, Tektonidou M, Seshan S. Characterization of antiphospholipid antibody-associated nephropathy: an international survey of Renal Pathology Society Members [abstract]. Arthritis Rheumatol. 2020; 72 (supp1 10). https:// acrabstracts.org/abstract/characterization-of-antiphospholipidantibody-associated-nephropathy-an-international-survey-of-renalpathology-society-members/. Accessed December 25, 2020.

23. Sevim E, Unlu O, Andrade D, Banzato A, Tektonidou M, et al. First and recurrent thrombosis risk after 1897 patient-years of follow-up: prospective results from Antiphospholipid Syndrome Alliance for Clinical Trials and International Networking (APS ACTION) Clinical Database and Repository ("Registry") [abstract]. Arthritis Rheumatol. 2018; 70 (suppl 10). https://acrabstracts.org/abstract/ first-and-recurrent-thrombosis-risk-after-1897-patient-years-offollow-up-prospective-results-from-antiphospholipid-syndromealliance-for-clinical-trials-and-international-networking-aps-actioncli/. Accessed December 25, 2020.

24. Sevim E, Andrade D, Banzato A, Tektonidou M, Ugarte A, et al. Pregnancy outcomes of antiphospholipid antibody positive patients: prospective results from Antiphospholipid Syndrome Alliance for Clinical Trials and International Networking (APS ACTION) Clinical Database and Repository ("Registry") [abstract]. Arthritis Rheumatol. 2018; 70 (suppl 10). https:// acrabstracts.org/abstract/pregnancy-outcomes-of-antiphospholipidantibody-positive-patients-prospective-results-fromantiphospholipid-syndrome-alliance-for-clinical-trials-andinternational-networking-aps-action-clinical-da/. Accessed December 25, 2020.

25. Tripodi A, Cohen H, Devreese KMJ. Lupus anticoagulant detection in anticoagulated patients. Guidance from the Scientific and 
Standardization Committee for lupus anticoagulant/antiphospholipid antibodies of the International Society on Thrombosis and Haemostasis. J Thromb Haemost. 2020;18(7):1569-75.

26. Devreese KMJ, de Groot PG, de Laat B, et al. Guidance from the Scientific and Standardization Committee for lupus anticoagulant/ antiphospholipid antibodies of the International Society on Thrombosis and Haemostasis. Update of the guidelines for lupus anticoagulant detection and interpretation. J Thromb Haemost. 2020;18:2828-39.

27. Pengo V, Denas G, Zoppellaro G, Jose SP, Hoxha A, Ruffatti A, et al. Rivaroxaban vs warfarin in high-risk patients with antiphospholipid syndrome. Blood. 2018;132(13):1365-71.

28. Ortel T. Anticoagulation withdrawal in in antiphospholipid syndrome. https://grantome.com/grant/NIH/U34-HL123499-01. Accessed December 25, 2020.

29. Barbhaiya M, Andrade D, Erkan D. AntiPhospholipid Syndrome Alliance for Clinical Trials and InternatiOnal Networking (APS ACTION): 5-year update. Curr Rheumatol Rep. 2016;18(10):64.

30. Whicher D, Philbin S, Aronson N. An overview of the impact of rare disease characteristics on research methodology. Orphanet J Rare Dis. 2018;13(1):14.

31. Dolfus H, Lévy N. Maladies rares, un champ de recherche "exceptionnel" [Rare diseases: an "exceptional" field of research. Interview by Catherine Holué]. Rev Prat. 2012;62(1):89-90.

32. Rodziewicz M, D'Cruz D, Gulliford M, Hazra N. The epidemiology of the antiphospholipid syndrome in the UK, 1990-2016 [abstract]. Arthritis Rheumatol. 2019; 71 (suppl 10). https://acrabstracts.org/ abstract/the-epidemiology-of-the-antiphospholipid-syndrome-in-theuk-1990-2016/. Accessed December 12, 2020.

33. Kearon C, Parpia S, Spencer FA, Baglin T, Stevens SM, Bauer KA, et al. Antiphospholipid antibodies and recurrent thrombosis after a first unprovoked venous thromboembolism. Blood. 2018;131(19):2151-60.

34. Miranda S, Park J, Le Gal G, Piran S, Kherani S, Rodger MA, et al. Prevalence of confirmed antiphospholipid syndrome in 18-50 years unselected patients with first unprovoked venous thromboembolism. J Thromb Haemost. 2020;18(4):926-30.

35. Garcia D, Akl EA, Carr R, Kearon C. Antiphospholipid antibodies and the risk of recurrence after a first episode of venous thromboembolism: a systematic review. Blood. 2013;122(5):817-24.

36. Ortel TL, Meleth S, Catellier D, Crowther M, Erkan D, Fortin PR, et al. Recurrent thrombosis in patients with antiphospholipid antibodies and an initial venous or arterial thromboembolic event: a systematic review and meta-analysis. J Thromb Haemost. 2020;18(9):2274-86.

37. Galli M, Luciani D, Bertolini G, Barbui T. Lupus anticoagulants are stronger risk factors for thrombosis than anticardiolipin antibodies in the antiphospholipid syndrome: a systematic review of the literature. Blood. 2003;101(5):1827-32.

38. Gebhart J, Posch F, Koder S, Perkmann T, Quehenberger P, Zoghlami C, et al. Increased mortality in patients with the lupus anticoagulant: the Vienna Lupus Anticoagulant and Thrombosis Study (LATS). Blood. 2015;125(22):3477-83.

39. Pengo V, Ruffatti A, Legnani C, Testa S, Fierro T, Marongiu F, et al. Incidence of a first thromboembolic event in asymptomatic carriers of high-risk antiphospholipid antibody profile: a multicenter prospective study. Blood. 2011;118(17):4714-8.

40. Sciascia S, Sanna G, Murru V, Roccatello D, Khamashta MA, Bertolaccini ML. Anti-prothrombin (aPT) and antiphosphatidylserine/prothrombin (aPS/PT) antibodies and the risk of thrombosis in the antiphospholipid syndrome. A systematic review. Thromb Haemost. 2014;111(2):354-64.

41. De Craemer AS, Musial J, Devreese KM. Role of anti-domain 1- $\beta 2$ glycoprotein I antibodies in the diagnosis and risk stratification of antiphospholipid syndrome. J Thromb Haemost. 2016;14(9):1779-87.
42. Arachchillage DR, Efthymiou M, Mackie IJ, Lawrie AS, Machin $\mathrm{SJ}$, Cohen $\mathrm{H}$. Anti-protein $\mathrm{C}$ antibodies are associated with resistance to endogenous protein $\mathrm{C}$ activation and a severe thrombotic phenotype in antiphospholipid syndrome. J Thromb Haemost. 2014;12(11):1801-9.

43. Zuo Y, Yalavarthi S, Gockman K, Madison JA, Gudjonsson JE, Kahlenberg JM, et al. Anti-neutrophil extracellular trap antibodies and impaired neutrophil extracellular trap degradation in antiphospholipid syndrome. Arthritis Rheum. 2020;72(12):2130-5.

44. Chaturvedi S, Braunstein EM, Yuan X, Yu J, Alexander A, Chen $\mathrm{H}$, et al. Complement activity and complement regulatory gene mutations are associated with thrombosis in APS and CAPS. Blood. 2020;135(4):239-51.

45. Sciascia S, Radin M, Ramirez C. Evaluation of novel assays for the detection of autoantibodies in antiphospholipid syndrome. Autoimmun Rev. 2020;19(10):102641.

46. Moritz CP, Paul S, Stoevesandt O, Tholance Y, Camdensanche J-P, Antoine J-C. Autoantigenomics: holistic characterization of autoantigen repertoires for a better understanding of autoimmune diseases. Autoimmun Rev. 2020;19(2):102450.

47. Anaya J-M, Leon KJ, Rojas M, et al. Progress towards precision medicine for lupus: the role of genetic biomarkers. Expert Rev Precis Med Drug Dev, 32, 119-135.

48. Lever E, Alves MR, Isenberg DA. Towards precision medicine in systemic lupus erythematosus. Pharmacogenomics Personalized Med. 2020;13:39-49.

49. Welter D, MacArthur J, Morales J, Burdett T, Hall P, Junkins H, et al. The NHGRI GWAS Catalog, a curated resource of SNP-trait associations. Nucleic Acids Res. 2014;42(Database issue):D1001-6.

50. Höglund J, Rafati N, Rask-Andersen M, et al. Improved power and precision with whole genome sequencing data in genome-wide association studies of inflammatory biomarkers. Sci Rep. 2019;9(1):1684.

51. Cohen H, Cuadrado MJ, Erkan D, Duarte-Garcia A, Isenberg DA, Knight JS, et al. 16th International Congress on Antiphospholipid Antibodies Task Force report on antiphospholipid syndrome treatment trends. Lupus. 2020;29(12):1571-93.

52. Amigo MC, Goycochea-Robles MV, Espinosa-Cuervo G, Medina G, Barragán-Garfias JA, Vargas A, et al. Development and initial validation of a damage index (DIAPS) in patients with thrombotic antiphospholipid syndrome (APS). Lupus. 2015;24(9):927-34.

53. Barbhaiya M, Erkan D. The optimal tool for assessment of organ damage in antiphospholipid syndrome. J Rheumatol. 2013;40(1):89.

54. Torricelli AK, Remia M, Ugolini-Lopes O, Bonfa E, Andrade D. Antiphospholipid syndrome damage index (DIAPS): distinct longterm kinetic in primary antiphospholipid syndrome and antiphospholipid syndrome related to systemic lupus erythematosus. Lupus. 2020;29(3):256-62.

55. Gaspar P, Cohen H, Isenberg D. The assessment of patients with the antiphospholipid antibody syndrome: where are we now? Rheumatology. 2020;59(7):1489-94.

56. Nantes SG, Strand V, Su J, Touma Z. Comparison of the sensitivity to change of the 36-item short form health survey and the lupus quality of life measure using various definitions of minimum clinically important differences in patients with active systemic lupus erythematosus. Arthritis Care Res. 2018;70(1):125-33.

57. Kahn SR, Hirsch A, Shrier I. Effect of post-thrombotic syndrome on health-related quality of life after deep venous thrombosis. Arch Intern Med. 2002;162(10):1144-8

Publisher's Note Springer Nature remains neutral with regard to jurisdictional claims in published maps and institutional affiliations. 


\section{Affiliations}

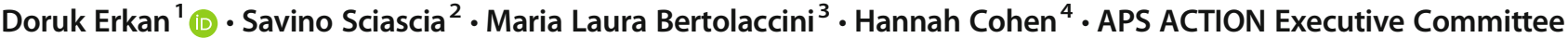

1 Barbara Volcker Center for Women and Rheumatic Disease, Hospital for Special Surgery, Weill Cornell Medicine, 535 E70th Street, New York, NY 10021, USA

2 Center of Research of Immunopathology and Rare Diseases, University of Turin, Turin, Italy

3 Academic Department of Vascular Surgery, King's College London British Heart Foundation Centre of Excellence, School of Cardiovascular Medicine \& Sciences, St Thomas' Hospital, London, UK

4 Haemostasis Research Unit, Department of Haematology, University College London, London, UK
5 University of Sao Paulo, Sao Paulo, Brazil

6 University of Hokkaido, Sapporo, Japan

7 University of Milan, Milan, Italy

8 Johns Hopkins University School of Medicine, Baltimore, MD 21205, USA

9 University of North Carolina, Chapel Hill, NC 27599, USA

10 National and Kapdistrian University of Athens, Athens, Greece 